\title{
State-of-the-Art von Smart Glasses zur Unterstützung von Logistikprozessen
}

Lucas Hüer, Benedikt Zobel, Hendrik Birkel und Oliver Thomas

Im Projekt GLASSHOUSE wird der praktische Einsatz von Smart Glasses als innovativer Technologietreiber im Dienstleistungsbereich der Logistik untersucht. $\mathrm{Zu}$ Beginn des Projekts sind noch nicht viele praktische Untersuchungen dieser Technologie in der zu untersuchenden Domäne erfolgt oder veröffentlicht. Um dennoch einen anfänglichen Wissens- und Kenntnisstand als Ausgangsbasis für weiterführende Untersuchungen benutzen zu können, wurde ein State-of-the-Art der Erkenntnisse aus Praxis und Wissenschaft mit der Anwendung von Smart Glasses im Logistiksektor erhoben. In diesem Beitrag werden Vorgehen und Ergebnisse dieser Initialuntersuchung präsentiert, gefolgt von einer Herleitung praktischer Anwendungsfälle für Smart Glasses entlang der logistischen Wertschöpfungskette.

\section{Einleitung}

Der Dienstleistungsbereich der Logistik stellt in der heutigen Zeit eine zentrale Säule der deutschen Wirtschaft dar, die durch immer weiter steigende Kundenanforderungen und zunehmende Vernetzung stetig an Bedeutung gewinnt. Durch dieses Wachstum weist die Logistik allerdings auch spezifische Herausforderungen auf. So sind Prozesse, bspw. zum Warenumschlag, durch komplexe und kleinschrittige Arbeitsabläufe gekennzeichnet. Zusätzlich ist die logistische Wertschöpfung sehr personalintensiv und die Mitarbeiter müssen auf Lagerflächen mobil und flexibel bleiben. Um dem wachsenden Kostendruck und Preiskampf begegnen zu können, sind Effizienzsteigerungen erforderlich (Arnold et al. 2008). Zur Erreichung dieser befindet sich die Logistik im schnellen technologischen Wandel, mit verschiedenen Geräten und Innovationen, welche die Mitarbeiter bei ihren Arbeitsprozessen unterstützen sollen.

Eine innovative Technologie zur Unterstützung der Logistik stellen die 2012 erstmals durch Google in ihrer aktuellen Form präsentierten Smart Glasses dar. Durch die Visualisierung von Informationen in das Blickfeld des Benutzers und Möglichkeiten zur händefreien Bedienung und Steuerung sowie die Mobilität 
durch kabellose Netzwerke und Akkus sind Smart Glasses gut für die in Logistikbetrieben und Lagern herrschenden Anforderungen geeignet.

Ziel des Konsortialforschungsprojekts GLASSHOUSE war es, den Einsatz von Smart Glasses in verschiedenen Teilbereichen der Logistik zu untersuchen, um mögliche nutzenbringende Einsatzszenarien zu identifizieren und prototypisch zu erproben. Zu Beginn des Projekts musste jedoch zunächst untersucht werden, wie der aktuelle Stand der Technik in Verbindung mit der Logistikbranche sowohl in Wissenschaft als auch Praxis ist. Daher wird in diesem Beitrag mit Hilfe einer systematischen Literaturrecherche und einer Erhebung von durchgeführten Projekten und aktuellen praktischen Studien untersucht, wie der State-of-the-Art von Smart Glasses in der Logistik zum Zeitpunkt des Projektbeginns ausgeprägt war.

Zur Erhebung, Dokumentation und Darstellung des State-of-the-Arts von Smart Glasses in Bezug zur Logistik und den daraus resultierenden Untersuchungen in GLASSHOUSE wird dieser Beitrag anhand der folgenden drei primären Forschungsfragen strukturiert:

- FF 1: Wie ist der State-of-the-Art von Smart Glasses in Logistikprozessen?

- FF 2: Welche Stärken und Schwächen hat der Einsatz von Smart Glasses?

- FF 3: Welche generellen Nutzungsmöglichkeiten können im Logistikbereich aufbauend auf dem State-of-the-Art identifiziert werden?

Diese Darstellung der einleitenden Motivation und der Forschungsfragen wird im Abschnitt 2 von einer Vorstellung der durchgeführten Forschungsschritte ergänzt. In Abschnitt 3 werden die Ergebnisse der Literaturrecherche anhand der einzelnen Phasen der logistischen Wertschöpfungskette strukturiert, gefolgt von einer Darstellung der aktuellen Forschungstrends in Bezug zur Technologie der Smart Glasses in Abschnitt 4. In Abschnitt 5 werden aus diesen Ergebnissen unterschiedliche Anwendungsgebiete für Smart Glasses in der Logistik hergeleitet. Den Abschluss des Beitrags bilden die Diskussion sowie ein zusammenfassendes Fazit mit einem Ausblick auf zukünftige Forschung.

\section{Methode}

Die Literaturrecherche dieses Beitrags basiert auf den Ansätzen zur strukturierten Literaturrecherche von Webster und Watson (2002) und vom Brocke et al. (2009). Wie von Budgen und Brereton (2006) vorgeschlagen, wurden verschiedene Aspekte zur Wahrung der Rigorosität beachtet. So wurde ein Überprüfungsprotokoll zur Spezifizierung der Forschungsfragen und -methoden erstellt, eine Suchstrategie zur Identifizierung einer größtmöglichen Menge an relevanter Literatur wurde definiert, diese Strategie wurde zur Nachvollziehbarkeit dokumentiert und schließlich wurden spezifische Ein- und Ausschlusskriterien formuliert und benannt.

Die Literatursuche wurde mit Hilfe zwei primärer Stufen durchgeführt. Zunächst wurde eine Stichwortsuche in fünf wissenschaftlich relevanten und aner- 
kannten bibliographischen Datenbanken durchgeführt. Eine Schlüsselwortmatrix wurde basierend auf einer Dimension für die Technologie und einer weiteren für den Anwendungsbereich erstellt. So wurde der Suchterm wie folgt festgelegt: („Smart Glasses” OR „Data Glasses” OR „Digital Eye Glasses” OR „Datenbrillen” OR „Augmented Reality” OR „Head-Mounted-Display” OR „HMD” OR „Head-Worn-Display” OR „HUD” OR „Wearable Device” OR „Wearable”) AND („Pick-by-Vision” OR „Logistik” OR „Warenwirtschaft” OR „Lagerhaltung” OR „Logistics” OR „Warehousing” OR „Supply Chain”). Es wurden nur Artikel ausgewählt, die in englischer oder deutscher Sprache verfasst wurden. Beiträge in weiteren Sprachen wurden von den Resultaten exkludiert. Initial konnten 3.145 Artikel identifiziert werden. Nach der Eliminierung von Dubletten und einer Überprüfung sowie Filterung auf die Relevanz von Titeln, Abstracts und darauffolgend dem Volltext wurden 14 Primärquellen für die weitere Analyse ausgewählt. Um aktuelle Forschungsprojekte, Praxisstudien und die Marktforschung einzubeziehen, wurde eine weitere Suche über allgemeine Suchmaschinen wie Google und die „Bielefeld Academic Search Engine“ (BASE) durchgeführt. Diese zweite Suche führte zu 84.800 ersten Ergebnissen, die dann auf 13 Primärartikel gefiltert werden konnten. In Kombination wurden so 27 Primärpublikationen erreicht und durch die Anwendung einer Vorwärts- und Rückwärtssuche um weitere 18 Quellen angereichert.

\section{Literatur zu Smart Glasses in der Logistik}

\subsection{Analyse der relevanten Literatur}

Um die Ergebnisse der Literaturrecherche verständlich zu erläutern, wurden alle gefundenen Artikel den verschiedenen Phasen eines Logistikprozesses zugeordnet. Folgende Prozessphasen wurden hierfür als Kategorien bestimmt: Wareneingang, Sortierung, Einlagerung, Kommissionierung und Warenausgang (Arnold et al. 2008). Da eine trenngenaue Einteilung nicht immer möglich ist, bedarf es einer Erweiterung der Kategorien in zwei zusätzliche Bereiche: „Weitere Einsatzmöglichkeiten“ und „Allgemeines“. Zur Kategorie „Weitere Einsatzmöglichkeiten“ zählen Aufgaben, die den herkömmlichen Logistikprozessen aufgrund ihrer Differenz nicht zugeordnet werden können. Zur Kategorie „Allgemeines“ werden Publikationen hinzugefügt, deren Forschungen auf einer Metaebene durchgeführt wurden und somit allgemeiner gehalten sind.

Für einen ersten Überblick wurde Literatur gemäß der verwendeten Definition der verwendeten Technologie sortiert. Beiträge, die die moderne Definition von Smart Glasses verwenden, konnten ab 2005 gefunden werden; durch die offizielle Einführung erster käuflich erhältlicher Geräte im Jahr 2012 nahm zu diesem Zeitpunkt die Zahl der relevanten Veröffentlichungen stark zu. Bis 2009 wiesen Beiträge häufig den heutzutage eher weniger benutzten Begriff der Head-MountedDisplays (HMDs) auf, nach 2009 sanken diese Veröffentlichungen allerdings bis 
2016 auf $0 \%$ ab. Insg. verwendeten $51 \%$ der identifizierten Publikationen primär HMDs, während die restlichen $49 \%$ die Anwendung moderner Smart Glasses beschrieben. Eines der produktivsten Forschungsteams in Verbindung mit Logistik war im Suchzeitraum ein Team von Forschern der Technischen Universität München, die den Begriff „Pick-by-Vision“ prägten. 31 \% der ausgewählten Publikationen stammen aus diesen Forschungsarbeiten. Da bei der Begriffsprägung von Pick-by-Vision klassische HMDs dem Stand der Technik entsprachen, wurden entsprechend ausschließlich HMDs in diesem Zusammenhang untersucht. $33 \%$ der gefundenen Veröffentlichungen wurden in Tagungsbänden gefunden, $27 \%$ in wissenschaftlichen Journalen und $16 \%$ in praktischen Magazinen und Zeitschriften. Die restlichen $24 \%$ entfielen auf Forschungsberichte, Abschlussarbeiten, Dissertationen, Bücher oder hochschuleigene Veröffentlichungen.

Die Publikationen wurden dann entsprechend ihrem Hauptfokus sortiert und den verschiedenen Phasen des Logistikprozesses zugeordnet. Durch die Kategorisierung konnten die 45 Publikationen insg. 64-mal zugeordnet werden. Dabei sind 31 Publikationen nur einer Kategorie zugeteilt (69\%), zehn Arbeiten zwei Kategorien (22\%), drei Veröffentlichungen drei Kategorien (7\%) und nur eine Publikation vier Kategorien (2\%). Bei der Auswertung der Forschungsschwerpunkte zeigt sich ein bestimmter Fokus der Arbeiten. $69 \%$ aller Publikationen beschäftigen sich mit dem Prozess der Kommissionierung. Die zweithäufigste Kategorie ist 'Allgemeines' mit $29 \%$, von denen $46 \%$ die Themen Sicherheitsbedenken und soziale Interaktion untersuchen. Weitere $31 \%$ der Publikationen beschäftigen sich mit der prozessbezogenen Informations-bereitstellung. An dritter Stelle mit $20 \%$ sind die 'Weiteren Einsatzmöglichkeiten', die wiederrum zu einem Anteil von 56 $\%$ die Montage in Kombination mit dem Auffinden von Gegenständen behandelt. Es folgen die Kategorien Bereitstellung und Warenausgang (9\%), Wareneingang und Anlieferung (9\%) sowie Lagerhaltung und Inventur (7\%) sowie Sortierung und Einlagerung (0\%). Somit wurde in keiner Primärquelle explizit auf die logistische Phase der Sortierung und Einlagerung eingegangen.

\subsection{State-of-the-Art in den verschiedenen Phasen eines Logistikprozesses}

\subsubsection{Kommissionierung}

Da $69 \%$ der gefundenen Literatur der Kommissionierung zugeordnet werden können, ist dies die einzige Phase, die im Folgenden in einem separaten Unterkapitel analysiert wird.

Unter Kommissionierung versteht man das Zusammenstellen einer Ware aus einem bereitgestellten Artikelsortiment nach vorgegebenen Aufträgen (Gudehus 2010). Der Prozess des Kommissionierens ist die arbeitsaufwändigste und kostenintensivste Aktivität in einem Warenlager und zugleich die schwierigste Aufgabe in der innerbetrieblichen Logistik (de Koster et al. 2007; Gudehus 2010). Die Komplexität des Kommissionierens ergibt sich aus der Vielzahl von Verfahren, 
Techniken und Kombinationsmöglichkeiten aus unterschiedlichsten Strategien, nach denen sich Kommissioniersysteme aufbauen und organisieren lassen (Gudehus 2010). Abhängig von dem industriellen Sektor betragen die Logistikkosten rund fünf bis neun Prozent der Gesamtkosten (Straube und Pfohl 2008). Davon entfallen wiederum rund $55 \%$ auf das Kommissionieren (Bartholdi und Hankman 2016). Der Mensch ist dabei ein wesentliches Element und kann aufgrund seiner Flexibilität nur schwer maschinell ersetzt werden (Reif und Günthner 2009). Durch den Faktor Mensch können allerdings auch Fehler auftreten. Diese beeinflussen die Qualität der Zustellung und dadurch auch die Beziehung zum Kunden negativ. Eine Minimierung oder möglichst vollständige Eliminierung der Fehler ist daher von größter Wichtigkeit. Die Kommissionierung wird in drei Teilschritte untergliedert: Vorbereitung, Kommissioniervorgang, Nachbereitung. Das Hauptaugenmerk der analysierten Studien liegt auf dem Kommissioniervorgang, da dieser als Haupthandlung den Weg zum Entnahmeort, die Entnahme und Ablage beinhaltet. Dies sind die wichtigsten und daher die zu optimierenden Vorgänge. Eine Fehlervermeidung ist aufgrund hoher Folgekosten von höchster Priorität (Rammelmeier et al. 2012). Die Unterstützung des Kommissionierers beim Teilschritt „Kommissioniervorgang“ nennt sich Pick-by-Vision. Dieser Begriff wurde vom Münchner Forschungsteam entwickelt (Günthner et al. 2009). In der Literatur existieren verschiedene Bezeichnungen für das gleiche System, wie „Pick-byHMD“ oder „Pick-by-HUD“. Für eine einheitliche Darstellung werden in dieser Arbeit alle Systeme unter dem Begriff „Pick-by-Vision“ zusammengefasst.

Pick-by-Vision bezeichnet ein Konzept „,zur Leistungs- und Qualitätssteigerung in der manuellen Kommissionierung durch die mobile, visuelle Informationsbereitstellung mit Hilfe innovativer Technologien aus den Bereichen AR und des Mobile Computing“ (Günthner et al. 2009, S. 3). Die Ausprägungen des Pick-by-Vision Systems sind sehr unterschiedlich und beinhalten mehrere, zu differenzierende Punkte. Sie reichen von einer einfachen visualisierten Kommissionierliste bis hin zur visuellen Navigation zum Warenbehälter. Von den insg. 45 Veröffentlichungen sind 31 der Kommissionierung zugeteilt worden. Diese beinhalten wiederum elf Studien, die den Einsatz des Systems in einem experimentellen Umfeld untersucht haben. Der Fokus der Studien liegt beim Kommissioniervorgang. Die Gemeinsamkeit aller Pick-by-Vision Systeme besteht darin, dass dem Nutzer Informationen in das Sichtfeld eingeblendet werden und so jederzeit verfügbar sind. Dadurch sind die Hände frei und der Kopf muss nicht fortlaufend gesenkt werden, um die Informationen von einer Papierliste oder einem Monitor abzulesen (Günthner und Rammelmeier 2012; Stocker et al. 2016; Theis et al. 2016). Die Form und Art der Informationsdarstellung ist vielfältig. Elementare Informationen, die in allen Studien betrachtet wurden, sind die Artikelnummer, die zu entnehmende Stückzahl sowie das Lagerfach (Rammelmeier et al. 2012). Weitere Möglichkeiten sind Abbildungen der zu entnehmenden Produkte oder 2D Darstellungen des Lagerhauses zur Orientierung (Günthner et al. 2009). Ein großer Unterschied entsteht durch die Verwendung eines Trackingsystems, das eine Darstellung von perspektivisch richtig überlagerten, virtuellen Informationen mit der 
Realität ermöglicht (Rammelmeier und Günthner 2014). Mithilfe des Trackingsystems können die Informationen z. B. in Form von Pfeilen oder Tunneln visualisiert werden, die den Kommissionierer durch das Lagerhaus navigieren oder den Ort des Regalbehälters markieren. Ein solches System wird allerdings nur in vier Studien beschrieben. Die Steuerung der Anzeige, also z. B. die Navigation durch das Menü, das Quittieren, Bestätigen und Weiterschalten zur nächsten Auftragsposition kann über einen Taster, eine Spracherkennung oder eine Gestensteuerung erfolgen (Günthner et al. 2009; Yamazaki et al. 2013; Mccall et al. 2015; Schlögl und Zsifkovits 2016). Neben den Funktionen zur Informationsdarstellung und Navigation kann die Kamera der Smart Glasses zum Scannen eines Barcodes genutzt werden. Dies ermöglicht im Kontext der Kommissionierung einen Abgleich der Kommissionierliste mit der entnommenen Ware, die Überprüfung der Korrektheit der entnommenen Menge der Ware und kann als automatische Weiterleitung zur nächsten Auftragsposition fungieren (Günthner und Rammelmeier 2012). Der Einsatz einer solchen Funktion wird jedoch nur in einer Studie realisiert (Günthner und Rammelmeier 2012).

Zur Unterstützung von Kommissionierern existieren neben Pick-by-Vision noch verschiedenste andere Methoden. $\mathrm{Zu}$ den verbreitetsten manuellen Kommissionierlösungen zählen Papierlisten, Pick-by-Voice, Pick-by-Light und Pick-byCMD (Cart-Mounted-Display). Eine Umstellung der Kommissioniermethode auf Pick-by-Vision wäre in der Praxis nur sinnvoll, wenn dadurch Optimierungen wie geringere Fehlerquoten und kürzere Bearbeitungszeiten realisiert werden, die die anderen Methoden nicht bieten. $\mathrm{Zu}$ den wichtigsten Verbesserungen zählen eine niedrigere Fehlerquote und eine kürzere Bearbeitungszeit der Aufträge, da diese direkte Auswirkung auf die Qualität der Leistung, Kundenbeziehung und Kosten haben (Reif und Walch 2008). Nachdem die gefundene Literatur verglichen wurde lässt sich feststellen, dass die Fehlerquoten der Kommissioniermethoden auf einem ähnlichen Level liegen. Nichtsdestotrotz erzielen die Pick-by-Vision Methoden sehr gute Resultate und weisen in acht der neun Studien geringere Fehlerquoten auf. Nur bei der Studie von Wu et al. (2015) ist die Fehlerquote 0,5\% höher als bei dem Pick-by-Light System. Hinsichtlich der Kommissionierdauer ist die Methode Pick-by-Vision in $67 \%$ der Studien schneller als die verglichenen Verfahren. Wie bei der Fehlerquote sind die Unterschiede allerdings gering und nur in den wenigsten Fällen signifikant. Im Allgemeinen kann gesagt werden, dass eine zusätzliche Überprüfung der Kommissionierung tendenziell zu weniger Fehlern, allerdings auch zu einer längeren Bearbeitungszeit führt.

\subsubsection{Weitere Einsatzmöglichkeiten in anderen Phasen des Logistikprozesses}

$\mathrm{Zu}$ den vorgeschlagenen Einsatzmöglichkeiten für die weiteren Logistikprozesse gab es in der analysierten Literatur keine Labor- und Praxisstudien; die Möglichkeiten werden daher im Folgenden nur theoretisch besprochen. So könnte zum Beispiel die Annahme und Identifikation der Güter im Wareneingang durch eine 
Datenbrille mit Scan-Funktion unterstützt werden (Dangelmaier et al. 2005; Mueck et al. 2005; Kückelhaus 2015). Zusätzlich kann eine Kontrolle sicherheitsrelevanter Artikel durchgeführt werden, die durch das Einblenden durchzuführender Arbeitsschritte sowie Hinweise auf potentielle Fehlerquellen unterstützt wird (Günthner et al. 2011). Zur Unterstützung der Lagerhaltung und Inventur kann die Smart Glasses mithilfe des Warehouse-Management-System (WMS) automatisch leere Behälter und Lagerplätze melden (Mueck et al. 2005; Iben et al. 2009). Dadurch werden auch andere Prozesse, wie z.B. die Kommissionierung unterstützt, da unterbrechungsfreie Prozessabfolgen geschaffen werden. Analog zur Kommissionierung kann eine Navigation, die Anzeige von Informationen zur Stückelung der Ware und der exakten Warenposition in der Lagerhaltung eingesetzt werden (Thomas et al. 2015). Bei der Bereitstellung und dem Warenausgang kann die Smart Glasses zur endgültigen Kontrolle eingesetzt werden. Da der Warenausgang die direkte Schnittstelle zum Kunden ist, werden in diesem Bereich aufwändige Überprüfungen durchgeführt (Rammelmeier et al. 2012). Analog zum Wareneingang kann die versandbereite Ware auf Richtigkeit und Vollständigkeit geprüft werden. (Dangelmaier et al. 2005; Mueck et al. 2005). Eine mögliche Ausprägung zur Vereinfachung des Prozesses könnte eine Visualisierung des Inhalts zur schnellen Überprüfung sein (Mueck et al. 2005). Eine weitere Einsatzmöglichkeit ist die Hilfe beim Lastmanagement, bei dem die Beladung von Transportmitteln unterstützt wird. Denkbar ist eine Anzeige zur optimalen Positionierung der Güter im Transportmittel (Krebs 2015; Thomas et al. 2015).

Zudem wurden weitere Einsatzmöglichkeiten analysiert, welche jene Anwendungen beschreiben, die den identifizierten Logistikprozessen nicht direkt zugeordnet werden können. Hierzu wird unter anderem ein Einsatz der Smart Glasses zur Unterstützung von Flurfahrzeugen gezählt. Die Fahrer können die Smart Glasses für folgende Einsatzszenarien nutzen: Navigationsunterstützung, Kollisionswarnungen, Anzeige des Betriebszustandes, Einblendung der Auftragsdaten und Markierung des Lagerplatzes (Günthner et al. 2015). Ein weiteres Einsatzszenario ist die Lagerhausplanung. Die Datenbrille kann beim Gang durch das Warenlager geplante Änderungen direkt einblenden. Dadurch können kritische Stellen wie herausragende Kanten einfach und schnell identifiziert und behoben werden (Reif und Walch 2008; Kückelhaus 2015). Auch mobile Trainings über Smart Glasses sind auf Grund der hohen Leiharbeiterquote in der Logistik notwendig (Reif und Walch 2008; Jörgl 2015). Die Smart Glasses kann zur Einführung bzw. zum Training neuer sowie bestehender Mitarbeiter genutzt werden, um die Einarbeitungszeit zu verkürzen. Dies bewirkt gleichzeitig eine Steigerung ihrer Qualifikation (Reif 2009; Hao und Helo 2015). Zusätzlich sind die Mitarbeiter dadurch selbstständiger, sodass sie kürzer begleitet und eingelernt werden müssen (Reif 2009).

\subsubsection{Ausgewählte Praxisbeispiele}

Die Firma DHL hat bereits gegen Ende 2014 im Rahmen eines Pilotprojekts den Einsatz von SGs in einem niederländischen Distributionszentrum getestet (DHL 
2015). Der Kommissionierprozess konnte laut Pressemitteilung um 25 \% verbessert werden. Der Zeitrahmen der Studie betrug drei Wochen, in denen zehn Kommissionierer mehr als 20.000 Warenposten sammelten und mehr als 9.000 Bestellungen erfüllten (DHL 2015). Die Ausrüstung bestand ausschließlich aus Smart Glasses, das heißt es wurden keine zusätzlichen in der Hand gehaltenen Scanner oder Picklisten benötigt (DHL 2015). Die verwendeten Modelle waren die Google Glass und die Vuzix M100, die die von Ubimax erstellte Software „xPick“ nutzten (DHL 2015). In dem von DHL veröffentlichten Bericht „Augmented Reality in Logistics“ von 2014 werden darüber hinaus mögliche Einsatzszenarien erarbeitet, die von der AR unterstützt werden könnten. Diese werden in vier Gruppen eingeteilt: Warenlageroptimierungen, Transportoptimierungen, die letzte Meile der Lieferung (Last Mile Delivery) und verbesserte Dienste mit Zusatznutzen (Value Added Services) (Glockner et al. 2014). Beispiele für Einsatzmöglichkeiten in den einzelnen Bereichen sind Pick-by-Vision, Warenlagerplanung, Vollständigkeitschecks von Ladungen, Navigation sowie die Unterstützung beim internationalen Handel durch Scannen und Übersetzten von Handelspapieren (Glockner et al. 2014). Nach dem ersten Pilotprojekt sollten die Datenbrillen gegen Ende 2015 auch in zwei US-Logistikzentren implementiert werden (Brandl 2015). Laut einer Pressemitteilung vom 31.08.16 beabsichtigte DHL, das Augmented-RealityProgramm mit Datenbrillen in den kommenden sechs Monaten auf weitere Branchen auszuweiten (DHL 2016).

Auch der Automobilhersteller Volkswagen hat Mitte 2015 ein dreimonatiges Pilotprojekt mit Datenbrillen durchgeführt. Die Pressemitteilung vom 23.11.2015 kündigte darauffolgend einen Serieneinsatz von Smart Glasses für die Kommissionierung in der Werkslogistik an (Volkswagen AG 2015). Das Projekt erfolgte auf freiwilliger Basis und umfasste 30 Mitarbeiter in verschiedenen Bereichen wie dem Einbau von Frontscheiben oder Gelenkwellen (Volkswagen AG 2015). Die Vorteile der Brillen sind laut VW die Einblendung notwendiger Informationen, eine einfache Handhabung, freie Hände, schnelle Einarbeitungszeiten neuer Mitarbeiter sowie eine erhöhte Prozesssicherheit (Volkswagen AG 2015).

\subsection{Herausforderungen bei der Adoption von Smart Glasses}

Der Einsatz von Smart Glasses in den verschiedenen Phasen des Logistikprozesses bietet neben den beschriebenen Vorteilen auch einige Herausforderungen, die analysiert und überwunden werden müssen. Zu den Herausforderungen gehören unter anderem die psychische und physische Belastung sowie die Akzeptanz der Nutzer und deren Sicherheitsbedenken.

Die psychische und physische Belastung durch Datenbrillen ist ein zentrales Thema, da diese je nach Ausprägung eine Einführung im industriellen Umfeld verhindern können. In diesen Punkten muss zwischen Smart Glasses und HMDs differenziert werden, da große Unterschiede im Gewicht der Geräte vorliegen. Gesundheitliche Beschwerden wie Kopf- und Nackenschmerzen können durch die Nutzung leichterer Smart Glasses eliminiert werden. Ein repräsentatives Beispiel 
ist die Publikation von Theis et al. (2016), die ihre Studie mit HMD und Smart Glasses durchgeführt haben. Die Ergebnisse zeigen, dass Kopf- und Nackenschmerzen bei der Nutzung von Smart Glasses nicht auftreten. Der Tragekomfort ist höher, was zu einer niedrigeren Beanspruchung bei den Probanden führt (Wille et al. 2014; Theis et al. 2016). Allerdings ist die subjektive Beanspruchung für die Nutzer höher als bei Einsatz des Tablet-Computers (Theis et al. 2016). Die objektive Beanspruchung hingegen weist nach einer Eingewöhnungsphase keinen signifikanten Unterschied auf (Theis et al. 2016). Weitere Studien weisen unterschiedliche Resultate auf. In der Studie von Tümler et al. (2008) liegen keine Unterschiede zwischen subjektiver und objektiver Beanspruchung im Vergleich zur Papierliste vor, was für die Datenbrille spricht. Auch bei den anderen Studien treten im Vergleich zu alternativen Kommissioniermethoden keine merklich höheren kognitiven Belastungen oder Unterschiede in Konzentration und Beschwerdesymptomatik auf (Günthner et al. 2009; Reif 2009; Schwerdtfeger et al. 2011; Ehmann et al. 2012). Einzig bei der Studie von Baumann et al. (2012) klagten die Probanden über ein schlechteres Sehvermögen, Augenschmerzen, Konzentrationsprobleme und Erschöpfung durch das Equipment. Zurückzuführen ist dies allerdings auch auf die insg. schlechte Akzeptanz des Systems. Nur einer der Probanden führte die Studie zu Ende und mit zunehmender Dauer waren die Beschwerden vollständig rückläufig (Baumann et al. 2012).

Für die erfolgreiche Einführung einer neuen Technologie in ein Unternehmen sind Mitarbeiter-Akzeptanz und Sicherheitsbedenken essenzielle Faktoren für einen Wettbewerbsvorteil (Motus et al. 2007). Daher sollte den Nutzern gerade in der Einführungsphase ein gewisses Maß an Entscheidungsfreiheit und Mitspracherecht eingeräumt werden, um Probleme zu identifizieren und zu beheben (Motus et al. 2007).

\section{Forschungstrends in der Smart-Glasses-Literatur}

Ob die Unterstützung durch Datenbrillen für einen Prozess sinnvoll ist, hängt von mehreren Faktoren ab. So ist zu evaluieren, welche Aspekte eines Prozesses zu unterstützen sind und welche Inhalte und Informationen dargestellt werden müssen.

In der Literatur konnten verschiedene Indikatoren für und gegen den Einsatz von Smart Glasses identifiziert werden. Zu den relevantesten Faktoren für den Einsatz zählen die Freihändigkeit, die Mobilität sowie der hohe Bedarf an Informationen, die kurzgefasst im Blickfeld des Nutzers dargestellt werden können, ohne dass dieser seinen Blick von der Primäraufgabe abwenden muss. Es betrifft besonders Prozesse die zeitkritisch sind, eine geringe Fehlertoleranz mit additiven Kontrollmechanismen aufweisen und in einer Umgebung mit hoher Produktvarianz durchzuführen sind. Weiter unterstützt die Smart Glasses Vorgänge wie die Navigation, das Auffinden von Orten bis hin zur Erkennung kleiner Gegenstände. 
Indikatoren, die gegen den Einsatz von SGs sprechen, sind vor allem die Verdeckung des Sichtfeldes, die mögliche Ablenkung und Beanspruchung durch das Tragen der SG sowie Akzeptanzprobleme. Weniger sinnvoll ist der Einsatz auch, wenn es sich um Routinetätigkeiten handelt, bei der Mitarbeiter stärker abgelenkt als unterstützt wird.

Tabelle 1. Indikatoren für bzw. gegen Einsatz von Datenbrillen (Ehmann et al. 2012)

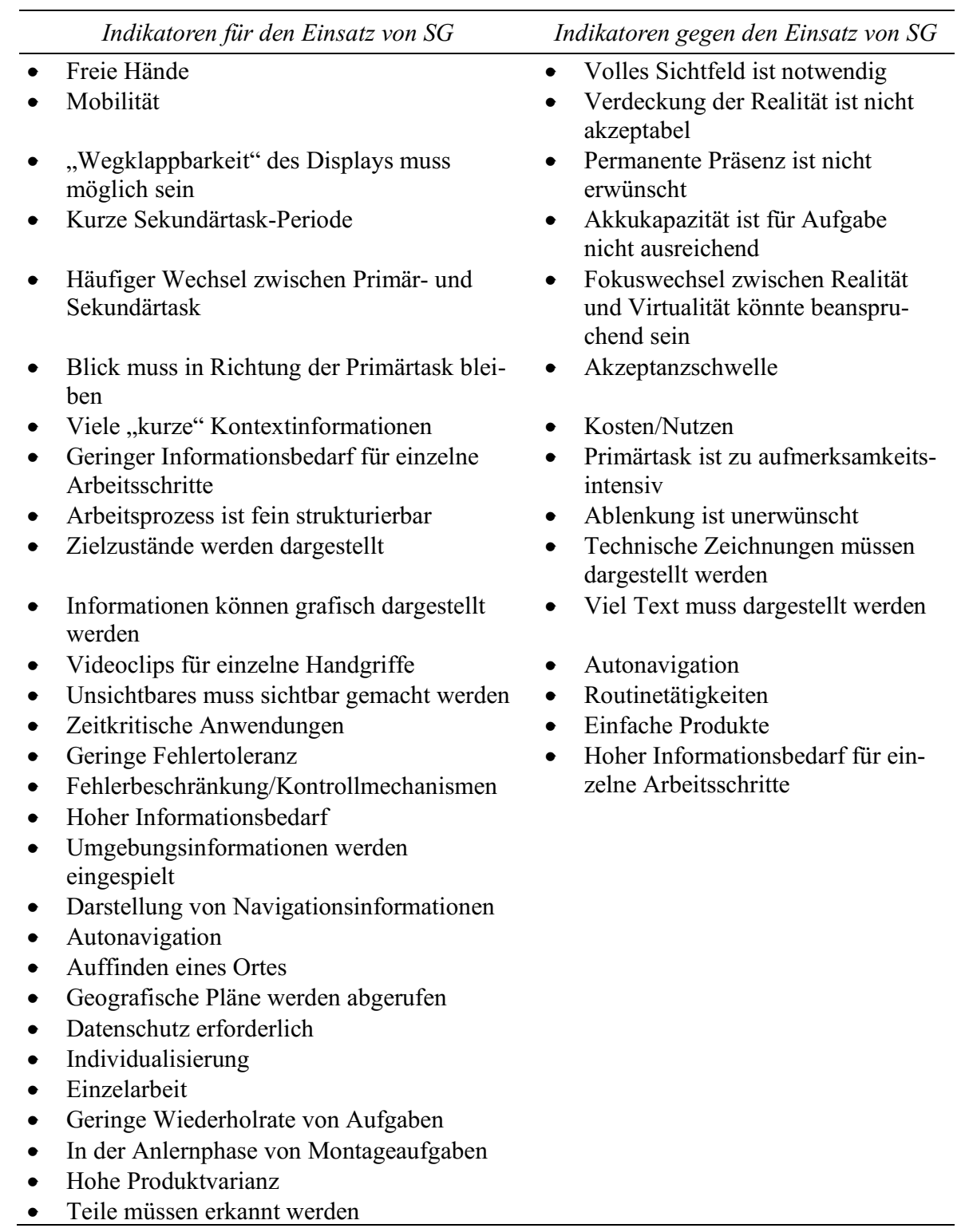




\section{Anwendungsgebiete für Smart Glasses in der Logistik}

Die neue Technologie der Smart Glasses wurde in vielen verschiedenen Bereichen der logistischen Wertschöpfung als Arbeitsmittel und als Innovation identifiziert. In diesem Abschnitt werden diese verschiedenen Anwendungsfelder und die zugehörigen technologischen Potenziale von Datenbrillen aufgezeigt.

\subsection{Wareneingang}

Beginnend bei der Warenannahme und der Anlieferung können Smart Glasses etwa durch eine schnelle Bearbeitung der Formalitäten die Arbeitsprozesse unterstützen. Durch das Scannen des Barcodes kann die Ware identifiziert, bestätigt und direkt im WMS vermerkt werden. Zudem versorgt die Datenbrille den Mitarbeiter mit Sicherheitshinweisen zum Umgang mit den Gütern und weist auf potenzielle Fehlerquellen bei der Kontrolle hin. Eine integrative neue Möglichkeit für eine reibungslose und effizientere Warenannahme könnte durch die direkte Kontaktaufnahme des Mitarbeiters mit dem Transportfahrzeugführer realisiert werden. So können notwendige Informationen wie Tornummern oder zu beachtende Hinweise schneller übermittelt werden. Diese Informationen können bspw. unmittelbar ins Blickfeld des Mitarbeiters eingeblendet und mit weiteren wichtigen Faktoren (bspw. verbleibende Dispositionszeit) angereichert werden. Darüber hinaus können Smart Glasses für ein verbessertes Wissensmanagement eingesetzt werden.

\subsection{Sortierung und Einlagerung}

Für die Prozesse im Bereich der Sortierung und Einlagerung konnten in den wissenschaftlichen Publikationen keine direkten Anwendungsfälle identifiziert werden. Durch einen hohen Grad der Automatisierung in diesem Teilbereich ist eine primär auf den Menschen ausgerichtete Unterstützung hier unter Umständen differenzierter zu betrachten. Sollte dieser Bereich in einem Unternehmen nicht automatisiert sein, könnten bewährte Funktionen aus der Kommissionierung in diesen Bereich übernommen werden. Beispiele sind die Kontrolle der einzulagernden Gegenstände durch Scannen der Barcodes, die Navigation durch das Warenlager sowie die Einblendung relevanter Informationen, wie Sicherheitshinweise, Handhabung oder Lagerort. Je nach Geschwindigkeit und Zuverlässigkeit des Scanvorganges können auch Pakete, die sich auf einem Förderband befinden, identifiziert und bei einem Fehler korrigiert werden.

\subsection{Lagerhaltung und Inventur}

Die Lagerhaltung kann ebenfalls von Smart Glasses unterstützt werden. Durch die registrierten Ab- und Zubuchungen bei der Entnahme und Einlagerung der Ware kann das WMS automatisch aktualisiert werden. Bei der Überprüfung der Lager- 
orte kann die Datenbrille dem Mitarbeiter den aktuellen Status naheliegender technischer Systeme melden. Zur optimierten Wegfindung ist eine Navigation möglich.

Bei der Inventur kann die Datenbrille dem Mitarbeiter automatisch den zu inventarisierenden Bereich markieren und anzeigen. Mithilfe der Scan-Funktion können die Lagerartikel registriert und im WMS verzeichnet werden. Es können bei Umlagerungen schnell freie Plätze identifiziert werden. Die Formulierung des Berichts der Inventur kann zudem über die Spracheingabe getätigt werden.

\subsection{Kommissionierung}

Insb. bei dem Anwendungsfeld Kommissionierung ist es wichtig, dass das Interface, also die Darstellung der Informationen, optimiert wird. Es muss anwendungsfallspezifisch geklärt werden was, wie, wann und wo angezeigt wird. Zum Beispiel kann sich die Darstellung eines laufenden Kommissionierers grundlegend von der eines fahrenden Kommissionierers unterscheiden. Daher ist es wichtig, dass eine Adaption der Darstellung für die Prozesse entwickelt wird.

Eine Weiterentwicklung der bisherigen Kommissioniertätigkeit wird durch die Arbeit von Yamazaki et al. (2013) angestoßen. Bisher wird zur Kontrolle der richtig entnommenen Artikel die Kamera der Smart Glasses genutzt, um einen Code zu scannen und eine Verwechslung auszuschließen. Mit der in der Publikation beschriebenen Möglichkeit zur automatischen Erkennung der Hand kann sich dies ändern. So wird der Artikel nicht erst nach Entnahme, sondern direkt während dieser überprüft. Dem Kommissionierer kann so unmittelbar angezeigt werden, ob sich seine Hand zum richtigen Regalfach bewegt. Der Prozess wird dadurch vereinfacht und beugt Fehlern zum frühestmöglichen Zeitpunkt vor. Durch die Gewichtung des Kommissionierprozesses und den Investitionen in Verbesserungen wird diese Option in Zukunft verfügbar sein, um Beschädigungen zu vermeiden. Als eine kleine Optimierung des Pick-by-Vision könnten dem Kommissionierer bei der Ablage der Waren in den Transportbehälter Hinweise zur Sortierung eingeblendet werden. Eine nicht zu unterschätzende weitere Möglichkeit, Mitarbeiter $\mathrm{zu}$ ermutigen und zu motivieren, besteht im Feedback. Nach geleisteter Arbeit könnte dem Mitarbeiter eine positive Bestätigung durch die Smart Glasses ausgegeben werden. So kann sowohl positives als auch negatives Feedback zu einer Steigerung der Performance führen (Fishbach et al. 2010). Im Falle eines Mitarbeiters in der Kommissionierung kann eine positive Rückmeldung als zielfördernd eingeschätzt werden. Auch dieses Feature kann in weitere Prozesse eingefügt werden um ein umfassend positives Arbeitsklima zu schaffen.

\subsection{Bereitstellung und Warenausgang}

Für die Bereitstellung von Waren und den Warenausgang können Smart Glasses zur Navigation, Identifikation und Kontrolle der zu versendenden Güter und zur Quittierung des Lieferauftrags genutzt werden. Sie können helfen, die Ware opti- 
mal in das Beförderungsfahrzeug zu verladen, indem die Reihenfolge und der Stellplatz visualisiert werden. Darüber hinaus kann das Gesamtgewicht der Ware mit den zulässigen Fahrzeugspezifikationen abgeglichen werden, um eine Überladung und damit die Gefährdung des Straßenverkehrs auszuschließen. Zusätzlich kann der Mitarbeiter des Warenlagers dem Fahrzeugführer Hinweise zur Handhabung der Ware zukommen lassen.

\subsection{Umfassende Anwendungsszenarien}

In Ergänzung dieser, jeweils auf einzelne Einsatzbereiche anwendbaren Beispiele, können weitere Potenziale bereichsübergreifend abgeschöpft werden. So können etwa Notizen per Spracherkennung erstellt und im WMS gespeichert werden. Angestellte können dadurch bspw. dazu motiviert werden, aktiver am Ideenmanagement zu partizipieren. Situationsbedingte Verbesserungen hinsichtlich einer Prozess- oder Arbeitsplatzoptimierung, die vom Mitarbeiter erkannt werden, können unmittelbar dokumentiert und in einem weiteren Schritt umgesetzt werden.

Ein weiteres übergreifendes Einsatzszenario ist eine Applikation zum Übersetzen fremder Sprachen. Vorrangig könnte diese Anwendung im Wareneingang bei der Interaktion mit Zulieferern eingesetzt werden. Eine Sprachbarriere kann so überwunden werden. Applikationen, die nahezu in Echtzeit übersetzen, sind schon vorhanden (Drees 2013; Horowitz 2014).

Die Autorisierung der eigenen Person, die an allen Arbeitsplätzen von Bedeutung ist, stellt einen anderen Anwendungsfall dar. So können durch das Abscannen von Codes und der Identifizierung die Zugangsmöglichkeiten zu speziellen Bereichen des Unternehmens geregelt werden. Dies kann auch vorrangig für die Unterscheidung von Mitarbeitern und Passanten dienen. Eine beispielhafte Anwendung dieses Szenarios könnte durch die Visualisierung eines virtuellen Buttons erfolgen, der nur dem Träger der SG sichtbar ist. Speziell im Bereich des Warenausgangs kann dies zum Öffnen eines Tors genutzt werden. Die Einsatzmöglichkeiten sind aber universell anwendbar und könnten flächendeckend zu einer Verminderung der zu installierenden Infrastruktur führen.

\section{Diskussion}

In Verbindung mit den verschiedenen im Rahmen dieses Beitrags untersuchten Forschungsfragen zeichnen sich auch Problembereiche oder Bedenken gegenüber der Technologie ab. Als Einsatzmöglichkeit von Smart Glasses wird in der Publikation von Günthner et al. (2015) der Einsatz bei Flurförderfahrzeugen aus Gründen der Sicherheit strikt abgelehnt. Das Beispiel des Einsatzes bei der Firma Dr. Babor zeigt allerdings, dass dies möglich ist. Da hier nur übliche, schnell zu erfassende Kommissionierangaben angezeigt werden, konnte ein Einsatz in diesem Feld Risiko-minimierend realisiert werden. Die Ablenkung des Fahrers ist nur minimal (WDR 2015). Bei Günthner et al. (2015) wurde stattdessen die Verwendung 
eines besser geeigneten Systems vorgezogen. Ein Einsatz bei Flurförderfahrzeugen stellt somit eine interessante Möglichkeit dar, bei der Aspekte der Sicherheit jedoch gesondert beachtet werden müssen.

Ein anderes mögliches Verwendungsszenario stellt die Planung von Lagerhäusern dar. Die Rentabilität dieser Möglichkeit wird durch einen sehr hohen Realisierungsaufwand allerdings als eher negativ eingeschätzt. Die Planung lässt sich effizienter und einfacher mit Computeranimationen oder unter Einsatz von VRTechnologien durchführen. Als weitere Applikationen kommen das Einlernen neuer Mitarbeiter sowie eine verbesserte, technologieunterstützte Kommunikation unter Mitarbeitern sowie zwischen Unternehmen und Kunden oder Lieferanten in Frage. Diese müssen allerdings in Zukunft genauer untersucht werden.

Neben den technologischen Möglichkeiten und wirtschaftlichen Rentabilitätsuntersuchungen sind psychische und physische Belastungen wichtige Faktoren, die für eine erfolgreiche Implementierung von Smart Glasses beachtet werden müssen. Die Publikationen zeigen, dass eine Verbesserung hinsichtlich Kopf- und Nackenschmerzen sowie des Tragekomforts von älteren, sehr schweren HeadMounted-Displays zu leichten und mobilen Smart Glasses erreicht wurde. Probleme wie Konzentrationsstörungen, Augenschmerzen oder Sehstörungen sind nach einer Eingewöhnungsphase ohne Weiteres spontan reversibel. Dies gilt auch für die subjektive und objektive Beanspruchung des Trägers. Die Datenbrillen gelten so als grundsätzlich für einen Einsatz in der Praxis geeignet. Weitere zentrale Untersuchungsaspekte stellen Akzeptanz und Sicherheitsbedenken gegenüber Smart Glasses dar. Die Studien weisen hierbei unterschiedliche Ergebnisse auf. Während bei Laborstudien die Motivation und Akzeptanz der Probanden positiv ausgeprägt sind, ist die Ablehnung in nur einer Praxisstudie von (Baumann 2013) erheblich. Die Berichte aus der industriellen Praxis zeigen eine positivere Akzeptanz. Ein Grund für die hohe Motivation bei Laborstudien kann durch die Probanden erklärt werden. Hierbei handelte es sich größtenteils um Studenten und Akademiker, die sich freiwillig und aus eigenem Interesse gemeldet haben. Akzeptanzprobleme sind unter diesen Bedingungen unwahrscheinlich.

Im Rahmen dieses Beitrags sollte der State-of-the-Art des Einsatzes von Smart Glasses in der Logistik untersucht werden, mit besonderem Fokus auf Einflussfaktoren und mögliche Einsatzgebiete. Da das Ziel der durchgeführten Literaturrecherche von wissenschaftlicher und praxisnaher Literatur im Erheben eines ,aktuellen" Standes zum Zeitpunkt des Projektbeginns lag, sind Forschungsaktivitäten und Erkenntnisse, die nach Beginn des Projekts begonnen oder durchgeführt wurden, nicht Teil dieser Studie.

\section{Fazit und Ausblick}

Bei der ausführlichen Literaturrecherche wurden insg. 45 Publikationen im Zeitraum von 2005 bis 2016 gefunden. 31 (69 \%) Publikationen betreffen das Kommissionieren. Bei den praktischen Studien für Logistikprozesse wurden alle in die- 
sem Einsatzgebiet durchgeführt. Dies liegt an der hohen Gewichtung, die der Kommissionierung zukommt. Zum einen entsteht dort ein Großteil der Lagerbetriebskosten (vgl. Abschnitt 3.2.1), zum anderen ist dies ein Bereich, in dem ein Großteil der Prozesse manuelle Tätigkeiten sind. Diese können durch Smart Glasses verbessert werden. Darüber hinaus deckt die Kommissionierung ein großes Spektrum an Prozessschritten ab, die in andere Logistikvorgänge übertragen werden können. Die Pick-by-Vision Systeme erreichen in 89 \% der Studien eine geringere Fehlerquote als andere Kommissioniermethoden. Die Fehlerquote bei Pick-by-Vision beträgt zwischen 0,12 \% und 1,25 \% mit einem Durchschnittswert von 0,64 \%. Die Durchschnittswerte der Fehlerquote der Papierliste (1,59\%), des Pick-by-Voice (3,05\%), des Pick-by-Light (2,10\%) und Pick-by-CMD (1,25\%) liegen höher. Auch bei der Kommissionierdauer führt das Pick-by-Vision-System in $67 \%$ der Studien zu einer schnelleren Bearbeitung der Aufträge. Dies zeigt das Potenzial der Pick-by-Vision-Systeme zur Optimierung der Kommissionierung deutlich.

Für die weiteren Logistikprozesse (neben der Kommissionierung) sind zusätzliche theoretische Einsatzmöglichkeiten gefunden worden. Diese beziehen sich auf die Bereiche Wareneingang, Lagerhaltung und Warenausgang. Grund dafür, dass der Bereich Sortierung und Einlagerung ausgeschlossen wird, liegt in der dort häufig bestehenden Vollautomatisierung. Die Möglichkeiten in den genannten drei Bereichen sind eine schnellere Warenannahme, die Visualisierung von Zusatzinformationen bei Gefahrengütern, die Unterstützung bei der Inventur und der Abschlusskontrolle sowie der Beladung von Transportfahrzeugen. Eine erleichterte Warenannahme kann durch das Scannen von Barcodes realisiert werden. Diese Funktion kann ohne Probleme in das Lagerhaus implementiert werden. Gleiches gilt für die Anzeige von Zusatzinformationen. Die Hilfestellung beim Beladen eines Transportfahrzeuges hingegen ist schwieriger zu realisieren. Die weiteren selbst entwickelten Einsatzmöglichkeiten (Use Cases) sind, nach Meinung der Autoren, sinnvolle Ergänzungen der bekannten Prozessschritte. Allerdings sind praktische Studien nötig, um den Grad einer Effizienzsteigerung zu testen. Für eine detaillierte Ermittlung weiterer Verwendungsmöglichkeiten ist die Zusammenarbeit mit einem Logistikdienstleister notwendig.

Aus den aufgeführten Ergebnissen ergeben sich neue Fragestellungen, welche durch weitere gezielte Forschungen beantwortet werden könnten. Eine der wichtigsten Aspekte ist der der mangelnden Forschungsstudien mit Smart Glasses. Im Optimalfall sollten die neuesten Smart Glasses Modelle eingesetzt werden. Eine weitere Identifikation von Einsatzmöglichkeiten in Zusammenarbeit mit einem Logistikunternehmen wäre interessant, um das Potenzial der Datenbrillen auszuschöpfen. Ein zusätzlicher Punkt, der in der Praxis beachtet werden muss, ist die rechtliche Grundlage der Überwachung des Mitarbeiters. Durch die Kommunikation der Smart Glasses mit dem WMS ist eine theoretische Kontrolle möglich. Es müssen Regelungen zum Umgang mit den Daten gefunden werden.

Abschließend stellt sich die Frage, für welche Logistikunternehmen eine Einführung von Smart Glasses interessant ist und wann diese gegebenenfalls stattfin- 
den sollte. Generell richtet sich die Technologie an mittlere und größere Firmen und Lagerhäuser, da eine Implementierung mit hohen Investitionen verbunden ist. Dazu zählen die Datenbrille, eine Adaption des WMS und Schulungen für das Personal. Aufgrund des Funktionsumfangs ist eine Einführung des Systems ohne Pick-by-Vision nicht sinnvoll. Angaben zu konkreten Kosten sind den Autoren nicht möglich. Sinnvoll ist ein Umstieg, wenn bisher nur Papierlisten oder ein Pick-by-Voice-System genutzt wurden. Ist ein Pick-by-Light-System installiert, welches mit hohen Investitionen verbunden war, ist ein Wechsel auf ein Pick-byVision-System nicht zu empfehlen.

\section{Literatur}

Arnold D, Isermann H, Kuhn A, Tempelmeier H, Furmans K (2008) Handbuch Logistik, 3. Ausgabe, Springer, Berlin, Heidelberg

Bartholdi J, Hackman S (2016) Warehouse \& distribution science, Atlanta

Baumann H (2013) Order picking supported by mobile computing. Dissertation, University of Bremen

Baumann H, Starner T, Zschaler P (2012) Studying order picking in an operating automobile manufacturing plant. In: Proceedings - International Symposium on Wearable Computers, ISWC. 112-113

Brandl, Nadine (2015) KEP: DHL setzt in den USA auf Datenbrillen. https://logistikheute.de/news/kep-dhl-setzt-den-usa-auf-datenbrillen-12044.html, abgerufen am 13.04.2016.

Budgen D, Brereton P (2006) Performing systematic literature reviews in software engineering. In: ICSE '06 Proceedings of the 28th international conference on Software engineering. 1051-1052

Dangelmaier W, Mueck B, Franke W (2005) Mixed Reality in Lagerprozessen. Verlagsschriftenreihe Heinz Nixdorf Institut 167:133-144

de Koster R, Le-Duc T, Roodbergen KJ (2007) Design and control of warehouse order picking: A literature review. European Journal of Operational Research 182:481-501. doi:10.1016/j.ejor.2006.07.009

DHL (2015) DHL successfully tests Augmented Reality application in warehouse. http://www.dhl.com/en/press/releases/releases_2015/logistics/dhl_successfully_tests_a ugmented_reality_application_in_warehouse.html, abgerufen am 21.06.2015

DHL (2016) DHL rollt globales Augmented Reality-Programm mit Datenbrillen aus. http://www.dpdhl.com/de/presse/pressemitteilungen/2016/dhl_rollt_globales_augment ed_reality-programm_aus.html, abgerufen am 01.09.2016

Drees $\bar{C}$ (2013) Vuzix M100 Smart Glass: App übersetzt Text direkt vor euren Augen. In: Mobilegeeks.de. https://www.mobilegeeks.de/vuzix-m100-smart-glass-app-uebersetzttext-direkt-vor-euren-augen/, abgerufen am 01.09.2016

Ehmann M, Wille M, Grauel B, Adolph L, Hoffmann P, Baumann H, Lawo M (2012) Datenbrillen - Aktueller Stand von Forschung und Umsetzung sowie zukünftiger Entwicklungsrichtungen. Bundesanstalt für Arbeitsschutz und Arbeitsmedizin, Dortmund, 23-28 
Fishbach A, Eyal T, Finkelstein SR (2010) How Positive and Negative Feedback Motivate Goal Pursuit. Soc Personal Psychol Compass 4:517-530. doi: 10.1111/j.17519004.2010.00285.x

Glockner H, Jannek K, Mahn J, Theis B (2014) Augmented reality in logistics. DHL Customer Solutions \& Innovation, Troisdorf

Gudehus T (2010) Logistik: Grundlagen - Strategien - Anwendungen. Springer, Berlin Heidelberg, 4. Ausgabe

Günthner WA, Blomeyer N, Reif R, Schedlbauer M (2009) Pick-by-Vision: Augmented Reality unterstützte Kommissionierung. In: Günthner WA (Hrsg) fml - Lehrstuhl für Fördertechnik Materialfluss Logistik. Technische Universität München, Garching

Günthner WA, Bengler K, vom Stein M, Knott V (2015) Einsatz der Augmented-RealityTechnologie zur Unterstützung des Fahrers von Flurförderzeugen, Abschlussbericht

Günthner WA, Rammelmeier T (2012) Vermeidung von Kommissionierfehlern mit Pickby-Vision. In: Günthner WA (Hrsg) fml - Lehrstuhl für Fördertechnik Materialfluss Logistik. Forschungsbericht, Technische Universität München, Garching

Günthner WA, Wölfle M, Fischer R (2011) Wearable Computing und RFID in Produktion und Logistik - Ansätze zur bereichsübergreifenden Nutzung digitaler Informationen. Logist J Nicht-Referierte Veröff, doi: 10.2195/1j_NotRev_guenthner_de_201110_01

Hao Y, Helo P (2015) The role of wearable devices in meeting the needs of cloud manufacturing: A case study. Robotics and Computer-Integrated Manufacturing 45:168-179

Horowitz BT (2014) 5 Must-Have Google Glass Apps For Road Warriors. In: cruxialcio.com. http://www.cruxialcio.com/5-must-have-google-glass-apps-road-warriors6541\#slide/5, abgerufen am 01.09.2016

Iben H, Baumann H, Ruthenbeck C, Klug T (2009) Visual based picking supported by context awareness. In: Proceedings of the 2009 international conference on Multimodal interfaces - ICMI-MLMI '09. 281-288

Jörgl T (2015) Jobs: Jeder fünfte Leiharbeiter in der Logistik tätig. http://www.logistikheute.de/Logistik-News-Logistik-Nachrichten/Markt-News/13167/GewaltigeUnterschiede-bei-Gehaeltern-im-Vergleich-zu-Stammbeschaeftigten-Jo, abgerufen am 01.09 .2016

Krebs D (2015) Supply Chain Agility : New Levels of Visibility through Mobile \& Wireless Investments. Supply Chain Management Review 19:40-46

Kückelhaus M (2015) Eleven reasons to consider augmented reality in logistics. Logistics \& Transport Focus 17:14

Mccall R, Popleteev A, Louveton N, Engel T (2015) Text entry on smart glasses A comparison of two input techniques. In: 8th International Conference on Human System Interaction (HSI), Warsaw, 195-200

Motus D, Boppert J, Walch D, Sauerland M, Hammerl M, Katzky U (2007) Informationskonservierung, -transfer und -bereitstellung. In: Günthner WA (Hrsg) Neue Wege in der Automobillogistik, Springer, Berlin, Heidelberg

Mueck B, Höwer M, Franke W, Dangelmaier W (2005) Augmented Reality applications for Warehouse Logistics. In: Abraham A, Dote Y, Furuhashi T, Köppen M, Ohuchi A, Ohsawa Y (Eds) Soft Computing as Transdisciplinary Science and Technology. Springer, Berlin, Heidelberg

Rammelmeier T, Galka S, Günthner WA (2012) Fehlervermeidung in der Kommissionierung. Logist Journal 2012:1-8. doi: 10.2195/1j_Proc_rammelmeier_de_201210_01

Rammelmeier T, Günthner WA (2014) Informatorische Prozesse in der manuellen Kommissionierung - Durchblick mit Datenbrille. Hebezeuge Fördermittel 54:28-30 
Reif R (2009) Entwicklung und Evaluierung eines Augmented Reality unterstützten Kommissioniersystems. Dissertation, Technische Universität München

Reif R, Günthner WA (2009) Pick-by-vision: augmented reality supported order picking. The Visual Computer 25:461-467. doi: 10.1007/s00371-009-0348-y

Reif R, Walch D (2008) Augmented \& Virtual Reality applications in the field of logistics. The Visual Computer 24:987-994. doi: 10.1007/s00371-008-0271-7

Schlögl D, Zsifkovits H (2016) Manuelle Kommissioniersysteme und die Rolle des Menschen. BHM Berg- Hüttenmänn. Monatshefte 161:225-228

Schwerdtfeger B, Reif R, Günthner WA, Klinker G (2011) Pick-by-vision: There is something to pick at the end of the augmented tunnel. Virtual Reality 15:213-223. doi: 10.1007/s10055-011-0187-9

Stocker A, Spitzer M, Kaiser C, Rosenberger M, Fellmann M (2016) Datenbrillengestützte Checklisten in der Fahrzeugmontage. Informatik-Spektrum 40:255-263

Straube F, Pfohl H-C (2008) Trends und Strategien in der Logistik. DVV Media Group $\mathrm{GmbH}$

Theis S, Pfendler C, Alexander T, Mertens A, Brandl C, Schlick C (2016) Head-Mounted Displays - Bedingungen des sicheren und beanspruchungsoptimalen Einsatzes. Bundesanstalt für Arbeitsschutz und Arbeitsmedizin, Dortmund

Thomas O, Özcan D, Zarvic N, Metzger D, Niemöller C (2015) Services @ digital - Innovation durch Smart Glasses. Service Today 4:36-37

Tümler J, Mecke R, Schenk M, Huckauf A, Doil F, Paul G, Pfister E, Böckelmann I, Roggentin A (2008) Mobile Augmented Reality in Industrial Applications : Approaches for Solution of User-Related Issues. In: IEEE International Symposium on Mixed and Augmented Reality

Volkswagen AG (2015) Volkswagen bringt 3D-Datenbrille in den Serieneinsatz. http://www.volkswagenag.com/content/vwcorp/info_center/de/news/2015/11/3D_smar t_glasses.html, abgerufen am 21.06.2016

vom Brocke J, Simons A, Niehaves B, Reimer K, Plattfaut R, Cleven A (2009) Reconstructing the Giant: On the Importance of Rigour in Documenting the Literature Search Process. In: ECIS 2009 Proceedings

WDR (2015) Datenbrille. In: ardmediathek.de. http://www.ardmediathek.de/tv/Lokalzeitaus-Aachen/Datenbrille/WDR-

Fernsehen/Video?documentId=30287990\&bcastId=7293556, abgerufen am 25.06.2016

Webster J, Watson RT (2002) Analyzing the Past to Prepare for the Future: Writing a Literature Review. MIS Quarterly 26(2):xiii-xxiii

Wille M, Scholl PM, Wischniewski S, Van Laerhoven K (2014) Comparing google glass with tablet-PC as guidance system for assembling tasks. In: Proceedings - 11th International Conference on Wearable and Implantable Body Sensor Networks, BSN Workshops 2014, 38-41

Wu X, Haynes M, Zhang Y, Jiang Z, Shen Z, Guo A, Starner T, Gilliland S (2015) Comparing Order Picking Assisted by Head-Up Display versus Pick-by-Light with Explicit Pick Confirmation. In: Proceedings IEEE International Symposium on Wearable Computers (ISWC). 1-4

Yamazaki K, Shibata F, Kimura A, Tamura H (2013) Prototype development of a mixed reality order picking system. Proc. Innovations in Information and Communication Science and Technology (ICST 2013), 15-24 